\title{
Production of computer-generated phase holograms using graphic devices: application to correlation filters
}

\author{
Andrés Márquez \\ Juan Campos, MEMBER SPIE \\ María J. Yzuel, MEMBER SPIE \\ Universitat Autonoma de Barcelona \\ Department de Física \\ E-08193 Bellaterra, Spain \\ E-mail: ifop8@cc.uab.es \\ Inmaculada Pascual, MEMBER SPIE \\ Universidad de Alicante \\ Departamento Interuniversitario de Óptica \\ Apartado 99 \\ E-03080 Alicante, Spain \\ Antonio Fimia, MEMBER SPIE \\ Universidad Miguel Hernández \\ Departamento de Ciencias Experimentales \\ y Tecnología \\ Avda. Ferrocarril s/n \\ Ed. Galia \\ 03202 Elche (Alicante), Spain
}

\author{
Augusto Beléndez, MEMBER SPIE \\ Universidad de Alicante \\ Departamento de Física \\ Ingeniería de Sistemas y Teoría de la Señal \\ Apartado 99 \\ E-03080 Alicante, Spain
}

\begin{abstract}
An available and low-cost method for the production of computer-generated phase holograms is presented. Firstly, binary masks are produced by a high-resolution graphic device. The factors that affect the fidelity of the holograms are analyzed. Secondly, enhanced light efficiency is obtained in copies made on holographic emulsions by bleaching them. The reproduction of low spatial frequencies, the usual ones with graphic devices, has been studied using diffraction gratings. We propose the super-Gaussian function to simulate the grating profile. The results obtained with that function present good agreement with the experimental results. The use of this model has enabled us to design a method that has been used to obtain the phase modulation in the bleached emulsion as a function of exposure. Through this study the bleached emulsion has been validated as a good phase material for low spatial frequencies, and has been applied for the production of optical correlation filters. We conclude that the bleached holographic filter improves the efficiency of the master with no significant loss of quality. (c) 2000 Society of Photo-Optical Instrumentation Engineers. [S0091-3286(00)02206-6]
\end{abstract}

Subject terms: computer-generated holograms; phase holograms; emulsion bleaching; thin gratings; low spatial frequencies; optical correlation filters.

Paper 980312 received Aug. 14, 1998; revised manuscript received July 8, 1999 , and Nov. 29, 1999; accepted for publication Dec. 6, 1999.

\section{Introduction}

Different technologies, such as lithography, single-point diamond turning, and laser and electron-beam pattern generators, can be used to record computer-generated holograms (CGHs). An overview of these technologies is in Ref. 1. The minimum feature size attainable with these techniques is approximately $0.5 \mu \mathrm{m}$, and this results in the production of good diffractive optical elements (DOEs) with high diffraction efficiencies. The quality and the detail of these DOEs are very good, and they permit one to manufacture fast optics and fine gratings. Nevertheless, these techniques are not easily available, because the equipment needed is very expensive. Furthermore, certain applications (optical correlation filters, ${ }^{2}$ interconnects ${ }^{3}$ etc.) do not need the production of very high-resolution holograms, and this permits the use of technologies with a minimum feature size larger than that in the technologies previously mentioned. An inexpensive alternative technique, based on high-resolution graphic devices ${ }^{2,4}$ is available to produce CGHs for this kind of applications. It is important to note that the quality of the holograms produced by a highresolution graphic device depends both on the resolution in the positioning of the printed point and on the size of the generated spot. ${ }^{5}$
Holograms produced on phase substrates have higher efficiency than holograms produced on absorption substrates. ${ }^{6}$ Making use of a copying process, it is possible to generate replicas on a phase substrate from an absorption hologram, which acts as a master. Vanin ${ }^{7}$ gives a review of the existing hologram copying processes. Pascual et al. ${ }^{8,9}$ show the application of these techniques to the production of holographic optical elements. In Refs. 3 and 10 these techniques are applied to the production of phase CGHs. The bleached photographic emulsion has been widely used as a phase substrate. It has a high resolution $(\approx 5000$ lines/ $\mathrm{mm}$ ), wide spectral range (400 to $700 \mathrm{~nm}$ ), low exposure energy $\left(\approx 100 \mu \mathrm{J} / \mathrm{cm}^{2}\right)$, and well-established processing techniques. A review of silver halide photographic emulsions applied to holography can be found in Ref. 11.

Computer-generated holograms are widely used in pattern recognition. ${ }^{12}$ In a previous work ${ }^{2}$ the application to optical correlation of computer-generated filters made with different techniques on absorption materials was studied. Campos et al. ${ }^{10}$ describe the generation of binary phaseonly filters (POFs) with the Burckhardt codification ${ }^{13}$ on a phase material by a copying technique; $649-\mathrm{F}$ Kodak plates were used. The master was generated with a laser printer and photoreduced over the holographic plate. In general the 
phase recording of a hologram introduces some distortions that cause deformation of the reconstructed object. ${ }^{14}$ However, when binary encoding is used, the impulse response of the binary phase-recorded filter is no more disturbed than the impulse response of the amplitude version. ${ }^{14}$ Therefore, the binary phase-recorded filter gives the same recognition capability as the amplitude-recorded filter.

In this paper we propose a technique for the production of computer-generated phase holograms. This technique involves the manufacture of binary masks by a highresolution graphic device. In order to increase the light efficiency, a contact print of these binary masks is made on a phase material.

In Sec. 2 the details of the manufacturing process are described. In order to calibrate the technique we generated several diffraction gratings with different frequencies from 4 to 32 lines $/ \mathrm{mm}$. These frequencies are low in comparison with gratings made by holographic interference. In general, a graphic device prints with a discrete positioning of the points and a given size of the spot. We study the limitations on the period and the symmetry of the gratings due to the discretization of the plotting device. As the final goal is the manufacture of phase holograms, once the master is produced, we make copies on a holographic material. These copies are bleached, and in the copying and bleaching process the symmetry of the phase gratings may change. We also study the dimensions and the symmetry of the phase gratings.

In Section 3 low-spatial-frequency gratings are analyzed in terms of their diffraction efficiency. The experimental results have been compared with the results calculated with the theoretical models that we propose to simulate the diffraction gratings. The dependence of phase modulation on exposure is empirically deduced by using these models in a three-step procedure.

In Sec. 4 we apply the proposed technique to the production of computer-generated holograms on bleached emulsion. We generate POFs and study their impulse response and their performance in a correlator setup for pattern recognition.

\section{Phase-Hologram Manufacture}

\subsection{Master Generation}

Digital techniques are used to design the diffraction gratings we are going to use in the copying process. The design is saved in a PostScript file, which is printed by a Linotronic 630 printer $^{15}$ on photographic film. This is a highresolution commercial laser writer usually used in graphic arts. A binary transmittance transparency of high contrast is produced. The fidelity of the implementation of the digital design depends on the resolution in the positioning of the spot and on the spot size printed by the graphic device. The resolution of the Linotronic 630 is 3251 dots per inch (dpi), which is equivalent to a separation $d=7.8 \mu \mathrm{m}$ between neighboring points. The diameter of the printed spot is $D$ $=13 \mu \mathrm{m}$. The combination of resolution and diameter of the spot limits the maximum spatial frequency that can be implemented on the transparency to approximately 30 lines $/ \mathrm{mm}$. To characterize and to optimize the technique, a series of binary gratings with different spatial frequency is generated. We define the symmetry factor $S$ as the ratio
Table 1 Values of the symmetry factors for the gratings. Here $N$ is the number of points in a period; $N_{D}$ is the number of dark points in a period; $S_{M}, S_{M}^{\prime}$, and $S_{B}^{\prime}$ are the symmetry factors of the digital design, of the master produced by the Linotronic printer, and of the bleached copy, respectively; and $\Delta$ is the difference between the clean-zone width in the bleached copy and the black-zone width in the master.

\begin{tabular}{rcrcccc}
\hline \hline$N$ & $\begin{array}{c}\text { Grating } \\
\text { (lines/mm) }\end{array}$ & $N_{D}$ & $S_{M}$ & $S_{M}^{\prime}$ & $S_{B}^{\prime}$ & $\begin{array}{c}\Delta \\
(\mu \mathrm{m})\end{array}$ \\
\hline 32 & 4 & 16 & 0.48 & 0.50 & 0.47 & 7.5 \\
16 & 8 & 8 & 0.46 & 0.46 & 0.41 & 6.3 \\
10 & 13 & 5 & 0.43 & 0.46 & 0.36 & 7.8 \\
7 & 18 & 3 & 0.48 & 0.49 & 0.41 & 4.4 \\
6 & $21 \mathrm{a}$ & 3 & 0.39 & 0.40 & 0.30 & 4.7 \\
6 & $21 \mathrm{~b}$ & 2 & 0.56 & 0.57 & 0.48 & 4.2 \\
5 & $26 \mathrm{a}$ & 2 & 0.47 & 0.45 & 0.31 & 5.5 \\
5 & $26 \mathrm{~b}$ & 1 & 0.67 & 0.75 & 0.60 & 5.9 \\
4 & 32 & 1 & 0.58 & 0.63 & 0.45 & 5.6 \\
\hline \hline
\end{tabular}

between the width of the dark zone and the width of the period. A perfectly symmetrical grating would correspond to $S=0.5$.

In general, we are interested in obtaining symmetrical gratings, because the more symmetrical the grating is the higher its efficiency. To this end we have generated the gratings specified in Table 1 . In column 1 the number $N$ of dots in a period is given. In column 2 the gratings are labeled. The number corresponds to the spatial frequency of the grating in lines per millimeter (to the nearest integer). In column 3 we show the number of dark points in a period, $N_{D}$, established in the digital design. For the gratings with 21 and 26 lines/mm two possibilities have been chosen. In column 4 the symmetry factor $S_{M}$ of the design, defined in terms of the size of the dots, is given. In column 5 we give the values of the symmetry factor $S_{M}^{\prime}$ measured experimentally on the masters generated by the Linotronic machine. This measurement is done on images captured with a CCD camera coupled to a microscope. Note that in Table 1 for the low-frequency gratings the value of the symmetry factor $S_{M}^{\prime}$ is close to 0.5 , while for the high frequencies $S_{M}^{\prime}$ is different from 0.5. In Fig. 1 images of four different master gratings are shown. We see the two zones of the binary absorption gratings.

\subsection{Phase Hologram}

The copying process used in this work consists in recording the pattern contained in the master in a holographic recording material. The master is placed in direct contact with the recording material, with the master and the photosensitive layer of the copy placed together. ${ }^{8}$ We use partially coherent light, which enables us to work with more economical sources and devices, and leads to stability conditions that are not as strict as those used in conventional holographic devices.

The binary gratings described in Table 1 are used as the masters in the copying process. The copies are made on 8E56 HD Agfa-Gevaert photographic emulsion. A collimated beam from a high-pressure mercury lamp, filtered at $405 \mathrm{~nm}$ and incident on the master and copy, is used to 


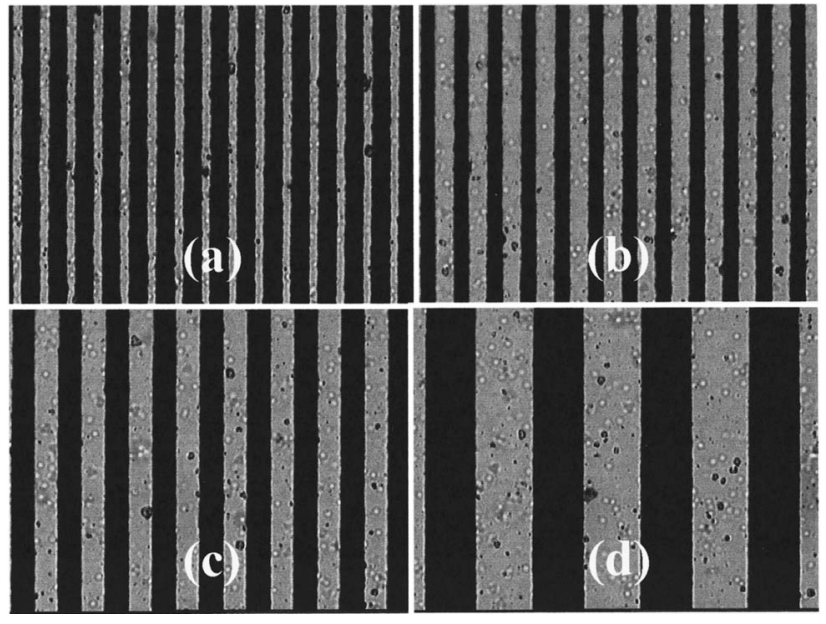

Fig. 1 Magnified images of master gratings of different frequencies made with the Linotronic printer. The frequencies are (a) 32 lines/ $\mathrm{mm}$, (b) 26 lines/mm (grating 26a), (c) 18 lines $/ \mathrm{mm}$, and (d) 8 lines/ $\mathrm{mm}$.

expose the photographic emulsion. In order to study the influence of the exposure we have produced several copied gratings for each frequency by varying the exposure between 10 and $425 \mu \mathrm{J} / \mathrm{cm}^{2}$. The exposed plates are developed in AAC developer, ${ }^{16}$ a nontanning developer made up of ascorbic acid and sodium carbonate. We have used a conventional rehalogenation bleach bath including potassium ferricyanide as oxidant. ${ }^{17}$ After the bleaching step, phase gratings are obtained.

In Fig. 2, different images of the phase gratings are shown. We distinguish a clean zone and a marked zone, corresponding respectively to the projection of the dark and transparent areas of the master. From the measurements done we find that the clean-zone width (corresponding to the black zone in the master) gets narrower in the copy in comparison with the black zone in the master. In column 6 of Table 1 we show the symmetry factor measured in the bleached copies, $S_{B}^{\prime}$, defined as the ratio between the width

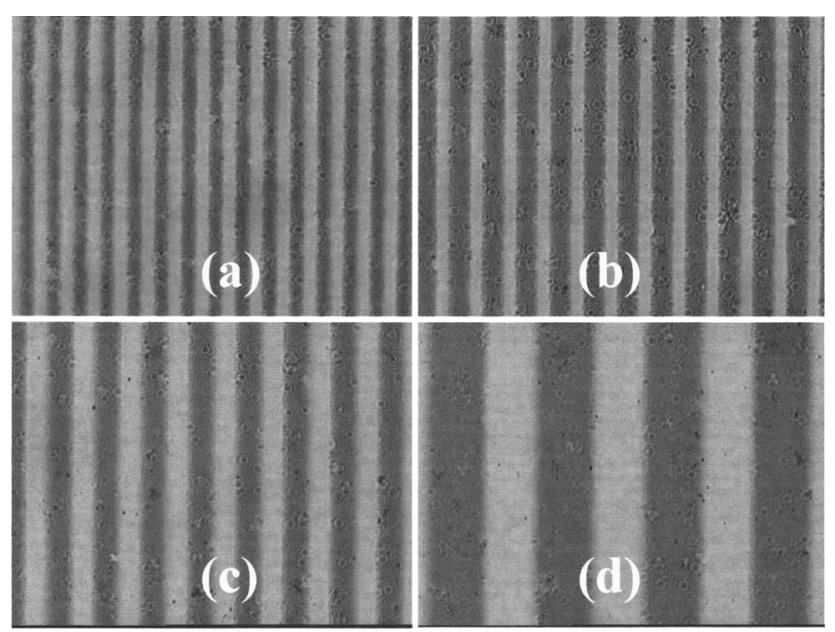

Fig. 2 Magnified images of copied and bleached gratings for different frequencies. The frequencies are (a) 32 lines $/ \mathrm{mm}$, (b) 26 lines/mm (grating 26a), (c) 18 lines $/ \mathrm{mm}$, and (d) 8 lines $/ \mathrm{mm}$.
Table 2 Minimum zero-order diffraction efficiency (DE0) and maximum first-order diffraction efficiency (DE1) values for the master gratings and for the bleached gratings (Exp: experimental values; SM: super-Gaussian model).

\begin{tabular}{|c|c|c|c|c|c|c|c|c|}
\hline \multirow[b]{3}{*}{$\begin{array}{l}\text { Grating } \\
(1 / \mathrm{mm})\end{array}$} & \multicolumn{4}{|c|}{ Master } & \multicolumn{4}{|c|}{ Bleached } \\
\hline & \multicolumn{2}{|c|}{ Exp } & \multicolumn{2}{|c|}{$\mathrm{SM}(\alpha=2)$} & \multicolumn{2}{|c|}{ Exp } & \multicolumn{2}{|c|}{$\operatorname{SM}(\alpha=1)$} \\
\hline & $\begin{array}{l}\text { DE0 } \\
(\%)\end{array}$ & $\begin{array}{l}\text { DE1 } \\
(\%)\end{array}$ & $\begin{array}{l}\text { DE0 } \\
(\%)\end{array}$ & $\begin{array}{l}\text { DE1 } \\
(\%)\end{array}$ & $\begin{array}{l}\text { DE0 } \\
(\%)\end{array}$ & $\begin{array}{l}\text { DE1 } \\
(\%)\end{array}$ & $\begin{array}{l}\text { DE0 } \\
(\%)\end{array}$ & $\begin{array}{l}\text { DE1 } \\
(\%)\end{array}$ \\
\hline 4 & 12.1 & 5.9 & 15.6 & 6.4 & 2.2 & 26.0 & 0.7 & 27 \\
\hline 8 & 12.7 & 5.5 & 15.6 & 6.4 & 3.6 & 25.1 & 0.2 & 27 \\
\hline 13 & 17.6 & 6.3 & 18.9 & 6.3 & 8.1 & 25.2 & 0.02 & 27 \\
\hline 18 & 18.2 & 6.8 & 15.6 & 6.4 & 7.3 & 25.6 & 0.2 & 27 \\
\hline $21 a$ & 21.9 & 6.3 & 22.5 & 6.0 & 0.1 & 20.5 & 0.08 & 27 \\
\hline $21 b$ & 9.9 & 5.3 & 10.0 & 5.7 & 8.6 & 23.0 & 2 & 27 \\
\hline $26 a$ & 16.9 & 6.5 & 18.9 & 6.3 & 6.4 & 25.1 & 0.08 & 27 \\
\hline $26 b$ & 3.1 & 2.3 & 3.9 & 3.1 & 12.6 & 18.6 & 7 & 24 \\
\hline 32 & 6.6 & 4.6 & 7.6 & 5.0 & 5.9 & 23.9 & 0.7 & 27 \\
\hline
\end{tabular}

of the clean zone and the width of the period. In column 7 we give the difference $\Delta$ between the width of the clean zone in the copy and the width of the dark zone in the master. This can be obtained as the product of the difference in the symmetry factors and the period: $\Delta=\left(S_{M}^{\prime}\right.$ $\left.-S_{B}^{\prime}\right) P(\mu \mathrm{m})$. The value of $\Delta$ varies between 4 and $8 \mu \mathrm{m}$.

\section{Diffraction Efficiencies in Gratings with Low Spatial Frequencies}

In this section the performance of the diffraction gratings described in Sec. 2 is evaluated in terms of diffraction efficiency. For the experimental measurement we have illuminated the grating with a plane wave from a $\mathrm{He}-\mathrm{Ne}$ laser $(\lambda=632.8 \mathrm{~nm})$ entering normal to the surface of the photographic emulsion. The diffracted beam intensity is measured, and the first-order diffraction efficiency (DE1) is calculated as the ratio between the energy in the first diffracted order and the incident energy. An analogous definition is established for the zeroth-order diffraction efficiency (DE0). We multiply these values by 100 to obtain the percentage of the incident energy that arrives in each diffraction order.

\subsection{Master Gratings}

In Table 2 we give experimental values of DE1 and DE0 (second and third columns, respectively) obtained with the master gratings described in Table 1 . We find that the maximum DE1 values approach 7\%.

To obtain deeper insight into the profile of the generated gratings and especially into the sharpness of the edges, we have obtained numerically the diffraction-efficiency values for three different grating models: sinusoidal, binary, and super-Gaussian. By comparing the numerical results with the experimental ones we determine that the model that best fits the experimental results is the supergaussian. With this kind of functions we can represent nonsymmetrical gratings and take into account the smoothing of the edges. The mathematical expression for these functions is 
Márquez et al.: Production of computer-generated phase holograms ...

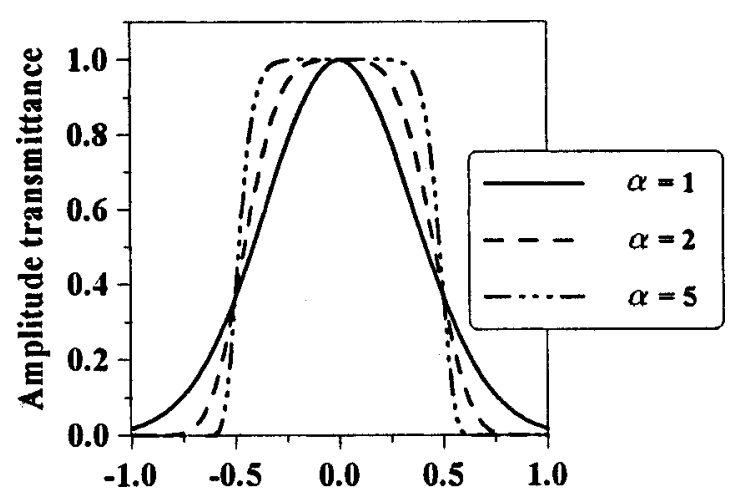

Fig. 3 Super-Gaussian function given by Eq. (3) for $t_{0}=1, \Omega=0.5$, and different $\alpha$ values.

$t(x)=t_{0} \exp \left[-\left(\frac{x}{\Omega}\right)^{2 \alpha}\right]$,

where $t(x)$ is the amplitude transmittance, $t_{0}$ is the maximum transmittance value, and $\Omega$ and $\alpha$ are parameters that characterize the function shape. The function is limited to an interval and repeated periodically. The value assigned to $\Omega$ permits the simulation of the symmetry factor, while $\alpha$ controls the sharpness of the edges (Fig. 3).

We have numerically evaluated the diffraction efficiencies for the super-Gaussian model with different $\alpha$ values from $\alpha=1$ to $\alpha=5$ and taking into account the experimental symmetry factors. The value of $\alpha$ that best fits the experimental results is $\alpha=2$. The corresponding values of the diffraction efficiency are given in Table 2, columns 4 and 5, labeled SM. There is good agreement between the experimental values of the diffraction efficiency and those obtained with this super-Gaussian function. This means that the gratings produced by the Linotronic have nearly vertical edges, as can be seen in Fig. $3(\alpha=2)$, but are clearly smoother than a perfectly binary grating profile.

\subsection{Bleached Gratings}

We made several copies of each master grating with different exposures. The exposure is the energy incident on the photographic emulsion per unit area in the copying process. The goal of this study, made by varying the exposure, is to obtain the highest DE1 value for each spatial frequency. In Fig. 4 we show the experimental curves of DE0 and DE1 versus exposure for bleached diffraction gratings of three different spatial frequencies. We observe that the DE1 curve attains one local maximum. From the different models (sinusoidal, binary, and super-Gaussian) we get that this first local maximum corresponds to a phase modulation of nearly $\pi$ rad in the emulsion. Approximately at the value of the exposure that corresponds to the first maximum of DE1, the DE0 curve attains its first local minimum. Every new DE1 local maximum implies an additional phase modulation of approximately $2 \pi$ rad. Therefore, from the curves presented we see that bleached emulsion reaches approximately $3 \pi$-rad phase modulation capability in the exposure range we have considered.

In Table 2 the experimental minimum for DE0 and the maximum for DE1 for each of the bleached gratings are
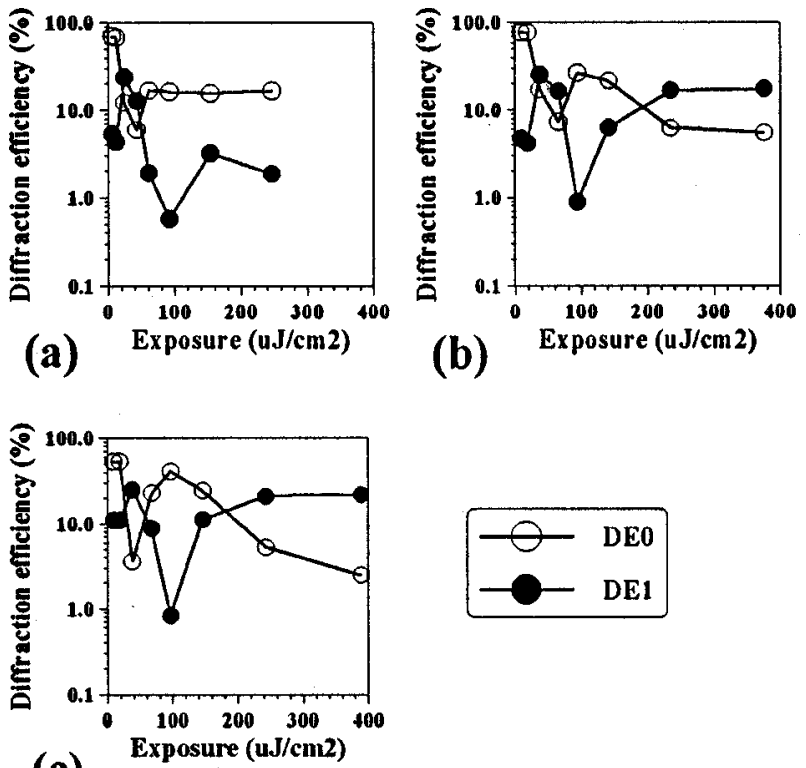

(c)

Fig. 4 Experimental DE curves (DE0 and DE1) for bleached gratings with different frequencies. (a) 32 , (b) 18 , (c) 8 lines $/ \mathrm{mm}$.

shown in columns 6 and 7, respectively. We have tried again to compare the experimental data with the values obtained by numerical simulation with the sinusoidal, the binary, and the super-Gaussian (SM) models for phase gratings. Again, the model that best fits the experimental values is the super-Gaussian, in this case the best parameter is $\alpha$ $=1$ (columns 8 and 9 in Table 2). The values of DE0 cannot be adjusted with any value of $\alpha$, but good agreement is found for DE1 values. As we have commented in Section 3.1 the masters follows the super-Gaussian model with $\alpha$ $=2$. This means that the copied gratings edges are smoother than the edges in the masters (compare the $\alpha$ $=1$ and $\alpha=2$ curves in Fig. 3). The fact that the bleached grating has less edge sharpness is due to both the copy step and the diffusion processes that transfer material from the exposed to the unexposed zones in the bleaching step.

Returning again to Table 2, we see that the experimental DE1 values can be as large as $26 \%$. This value is lower than the theoretical $33 \%$ maximum diffraction efficiency for a perfectly binary and symmetrical grating. But it is far higher than the $7 \%$ attainable with the master gratings. From column 7 in Table 2 we can also see that the maximum DE1 value does not present significant fluctuations in the spatial frequency of the bleached gratings. This enables us to state that conventional rehalogenation bleaching gives a high and frequency-independent diffraction efficiency for low-spatial-frequency holograms.

We also see that the experimental maximum DE1 values measured do not seem to be appreciably affected by the different symmetry-factor value of each grating, except for grating 26b, which is the only one that gives a value smaller than $20 \%$. That grating presents a symmetry factor $S_{B}^{\prime}=0.60$. If we look at column 9 , we see that the $\alpha=1$ super-Gaussian model predicts this behavior. For symmetry factors ranging from 0.31 to 0.48 the DE1 value is approximately the same $(27 \%)$, while the symmetry factor 0.60 


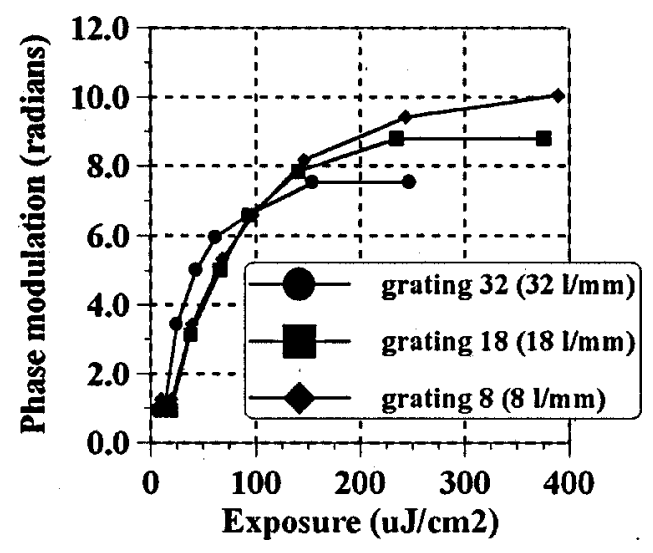

Fig. 5 Phase modulation as a function of exposure for three bleached gratings.

gives a lower value (24\%). We have also made the calculation for the symmetry factor $S_{B}^{\prime}=0.55$, obtaining DE1 $=26 \%$, and for $S_{B}^{\prime}=0.25$, obtaining DE $1=26 \%$. Then, according to the experimental data presented in Table 2 and to the super-Gaussian model with $\alpha=1$, bleached copies must present a symmetry factor higher than 0.25 to 0.30 and lower than 0.55 to 0.60 to maximize DE1. Therefore, for the maximum DE1 value there is a tolerance range in the symmetry factor. We notice that this tolerance range is not centered on a symmetry factor of 0.5 but it is biased to lower values.

\subsection{Phase Modulation as a Function of Exposure}

From the experimental curves shown in Fig. 4 and assuming that bleached gratings can be modeled with the superGaussian $\alpha=1$, we calculate the phase modulation $\phi_{0}(E)$ that best fits the experimental diffraction efficiency presented by the bleached gratings at each exposure value. To obtain the value of the phase modulation as a function of exposure we proceed as follows: For each grating we have the values of the diffraction efficiency as a function of the exposure $[\mathrm{DE} 1=\mathrm{DE} 1(E)]$. By using the super-Gaussian model with $\alpha=1$ we can obtain the diffraction efficiency as a function of the phase modulation $\left[\mathrm{DE} 1=\operatorname{DE} 1\left(\phi_{0}\right)\right]$. By comparing these equations we can obtain the phase modulation as a function of the exposure $\left[\phi_{0}=\phi_{0}(E)\right]$.

This method is capable of finding the curve $\phi_{0}$ $=\phi_{0}(E)$ by making use of the previous modeling of the gratings. In Fig. 5 we show the $\phi_{0}$-versus- $E$ curves corresponding to three different spatial frequencies $(32,18$, and 8 lines $/ \mathrm{mm}$ ). We see that for a phase modulation of $\pi \mathrm{rad \text {, }}$ which is when the curves attain the maximum DE1 value, the exposure in the three curves is very similar. We remark that the curves present a linear section up to a phase modulation of approximately $2 \pi \mathrm{rad}$. Then they tend to a saturation plateau.

The knowledge of these curves is especially important in the case where we want to register a hologram with a continuous phase profile, such as a kinoform. ${ }^{18}$ As the exposure received by the bleached emulsion in the copying step is proportional to the gray level, for the production of a kinoform we would need a mask with different gray levels.
In this way, taking into account the function $\phi_{0}=\phi_{0}(E)$, we can control the phases we introduce in the bleached emulsion by controlling the exposure value with the gray level of the mask.

As we have said, in the exposure range considered the phase modulation capability of the bleached emulsion is larger than $2 \pi$ rad. A $2 \pi$-rad capability is enough for any application of diffractive optics. Making use of the method to obtain the function $\phi_{0}=\phi_{0}(E)$, the technique proposed in this paper for binary phase holograms might be adapted for manufacturing multilevel phase holograms. A multilevel gray-tone graphic device should be used to generate the master.

\section{Computer-Generated Bleached Filters}

For each master grating we find that there is an exposure range, in the copying step, in which the bleached gratings produced have high diffraction efficiency. A generic hologram is composed of a combination of gratings of different spatial frequencies. Therefore, to copy a generic hologram an exposure value must be selected in the range of values that provide bleached gratings with high diffraction efficiency. When copying a generic hologram we are interested in the value of the exposure incident on the ensemble master copy, because in this way we do not need to know the particular transmittance of the generic hologram we will copy. From the experimental data $156 \mu \mathrm{J} / \mathrm{cm}^{2}$ seems to be a good value for the exposure incident on the master in the copying step.

\subsection{Performance of Bleached Filters}

Our interest is centered on the production of filters for pattern recognition by correlation methods. In the master we have encoded a phase-only filter (POF) by using Burckhardt's method. The filter is matched for the recognition of the lower butterfly in Fig. 6. In this paper we are interested in evaluating the proposed method to obtain phase holograms. Then any kind of encoding method can be used to produce the filter. We have selected Burckhardt's method because of its internal structure. Each cell is divided into three subcells and will give us local gratings with different periods. This means that the center-to-center distance can range between one-third and the total width of the cell Taking into account both directions, we can consider that the central frequency present in the hologram is due to the cell dimension. In the holograms we have made, each cell is a square of $12 \times 12$ points. This means a central frequency of 11 lines $/ \mathrm{mm}$. As we know, the highest spatial frequency attainable is about 32 lines $/ \mathrm{mm}$, so we can represent this type of hologram correctly with these dimensions.

For the production of the bleached filters we use the technique that we have proposed and applied for the production of gratings in Sec. 2 and 3. The exposure value used in the copying step is $156 \mu \mathrm{J} / \mathrm{cm}^{2}$. With this exposure we will obtain bleached filters with a high diffraction efficiency, which means that there is a phase modulation approximately equal to $\pi$ rad between the exposed and the unexposed zones in the copy.

The impulse responses of the master and of the bleached filter are then obtained (Fig. 7). We see that in the impulse response the edges of the butterfly are emphasized, i.e., the 


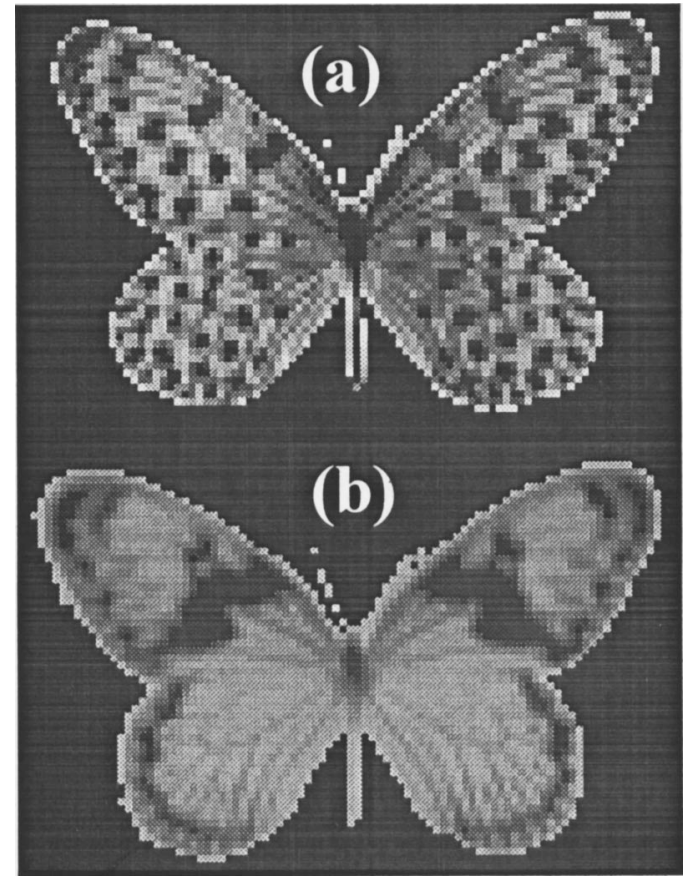

Fig. 6 Scene used for pattern recognition. Butterfly (b) is the target to which the filter is matched.

high frequencies. This is a consequence of the POF implementation. A significantly higher brightness is attained in the bleached filter's impulse response. In order to determine quantitatively the quality of the holograms, we have measured the energy per unit area, $E_{R}$, in the image reconstruction [reconstruction rectangle in Fig. 7(a)] in comparison with the energy per unit area, $E_{N}$, inside a rectangle between the reconstruction and the zeroth diffraction order [noise rectangle in Fig. 7(a)]. We take the ratio between $E_{R}$ and $E_{N}$ as a measure of the quality of the hologram, and call it the noise-reduction ratio (NRR): $\mathrm{NRR}=E_{N} / E_{R}$. Diffraction efficiency (DE) is defined as the intensity diffracted to the reconstructed butterfly divided by the incident intensity from the laser. In Table 3 NRR and DE values are shown. We see that diffraction efficiency in the copy is greater than $15 \%$. Therefore, good diffraction efficiency has been achieved with the selected exposure value. We

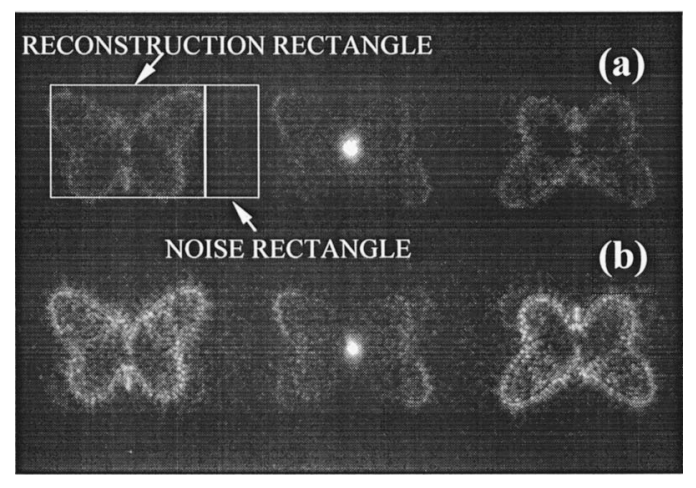

Fig. 7 (a) Impulse response of master filter, (b) bleached filter. In (a) reconstruction and noise rectangles are drawn.
Table 3 Performance of the filters in impulse response [noisereduction ratio (NRR) and diffraction efficiency (DE)] and in correlation [maximum autocorrelation peak energy and discrimination capability (DC)].

\begin{tabular}{lcccc}
\hline \hline Filter & NRR & $\begin{array}{c}\text { DE } \\
(\%)\end{array}$ & $\begin{array}{c}\text { Autocorrelation peak } \\
\text { (arbitrary units) }\end{array}$ & DC \\
\hline Master & 0.27 & 3.4 & 66 & 0.70 \\
Bleached & 0.17 & 16 & 200 & 0.65 \\
\hline \hline
\end{tabular}

also conclude that the diffraction efficiency (DE) is more than 4 times bigger than in the master $(3.4 \%)$. Besides, the NRR is better in the bleached copy.

The performance of the filters in a correlation setup (Fig. 8) has been examined experimentally. In Fig. 9 we show the correlation planes obtained with the master and the bleached filters. The scene is shown in Fig. 6. We have used the same scale in both Fig. 9(a) and Fig. 9(b). The autocorrelation peak is perfectly distinguishable from the cross-correlation peak in both cases. We have written in Fig. 9 the values of the autocorrelation peak. We also analyze the behavior of the filters in terms of the discrimination capability, defined as

$\mathrm{DC}=1-\frac{\text { maximum cross-correlation peak }}{\text { maximum autocorrelation peak }}$.

In Table 3, columns 3 and 4 , we give the values of the autocorrelation maximum and the DC for the master and the bleached CGH. We find that the bleached filter provides an autocorrelation peak with intensity three times higher than in the case of the master. The discrimination capability is slightly less for the bleached $\mathrm{CGH}$, but in both cases discrimination is ensured. We conclude that the phase filter is more efficient than the master and it has similar or even superior quality (a better NRR).

\section{Conclusions}

An available and low-cost technique for the production of computer-generated phase holograms has been proposed. For the calibration of the technique, diffraction gratings with different spatial frequencies have been produced. They are low-spatial-frequency gratings (from 4 to 32 lines $/ \mathrm{mm}$ ). We have used them to study the limitations introduced by the Linotronic printer in the generation of the master. On one side, the combination of the resolution in the positioning of the points and the diameter of the generated spot limit the maximum spatial frequency that can be registered (32 lines/mm with the Linotronic 630 we use). On the other side, consecutive points overlap each other, and the effect

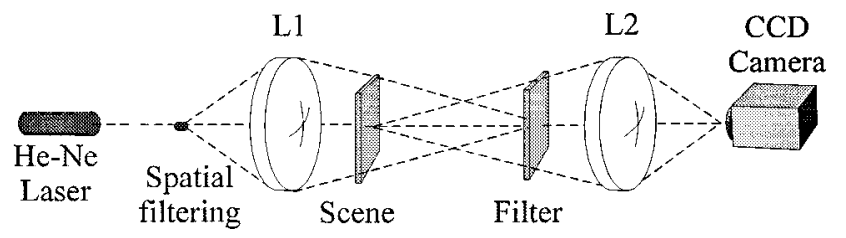

Fig. 8 Convergent correlator setup. 
(a)
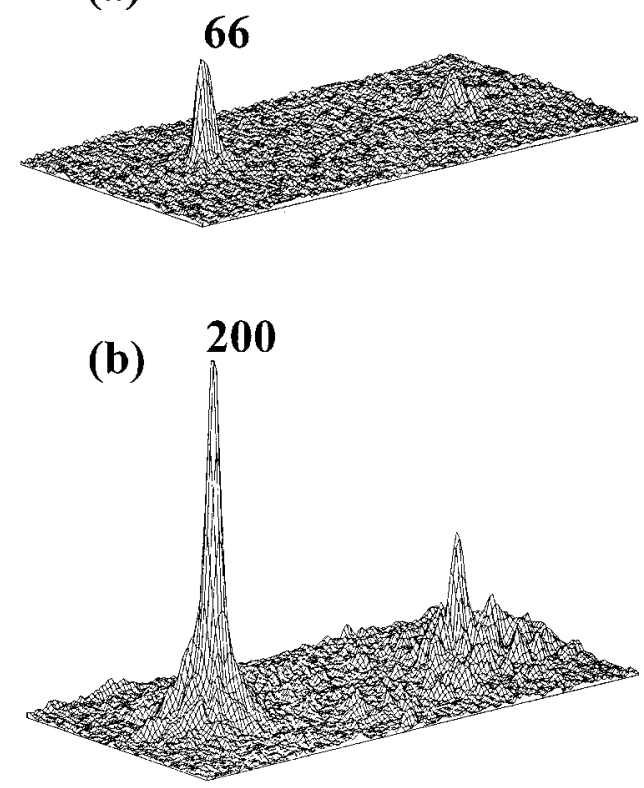

Fig. 9 Correlation planes obtained with (a) master filter, (b) bleached filter.

is a change in the dimensions of the digitally designed gratings when implemented on the transparency.

A strategy to obtain symmetrical gratings has been presented, and a study of the changes in the symmetry of the bleached gratings in comparison with the master gratings has been carried out. We have found that the dark-zone width in the master gratings narrows by 4 to $8 \mu \mathrm{m}$ in the bleached copies.

The generated gratings have also been studied with respect to the diffraction efficiency. We have compared the experimental diffraction-efficiency results with those provided by three different grating profile models. A model proposed by us, based on the super-Gaussian function, has been the one that best fits the experimental results.

A three-step method has been proposed to obtain the function that relates the phase modulation $\phi_{0}$ to the exposure $E$. In this method we make use of the experimental results on the diffraction efficiency and the values calculated with the models. This function is of interest when a multilevel phase hologram is registered.

The technique has been successfully applied for the production of computer-generated phase filters for optical correlation. They have a $16 \%$ diffraction efficiency, far higher than the $3.4 \%$ of the master. The NRR is lower in the bleached filter, and the DC is ensured. Therefore, in the bleached filters we gain efficiency without loss of quality.

\section{Acknowledgments}

A. Márquez wishes to thank the Comissionat per a Universitats i Recerca de la Generalitat de Catalunya for a grant. This work was partly supported by the Dirección General de Enseñanza Superior del Ministerio de Educación y Cultura (project PB96-1134-C02-01) and by CICYT (Comisión Interministerial de Ciencia y Technología) (project MAT97-0705-C02-02)

\section{References}

1. H. P. Herzig, Ed., Micro-optics-Elements, Systems, and Applications, Taylor \& Francis, London (1997)

2. I. Moreno, C. Gorecki, J. Campos, and M. J. Yzuel, "Comparison of computer-generated holograms produced by laser printers and lithography: application to pattern recognition," Opt. Eng. 34(12), 3520$3525(1995)$

3. A. Fimia, R. Fuentes, I. Pascual, A. Beléndez, J. J. Egozcue, and M. T. Navarro, "Copying computer-generated holographic interconnects by the use of partially coherent light," Appl. Opt. 33(8), 1431-1433 (1994).

4. D. C. O'Shea, J. W. Beletic, and M. Poutous, "Binary-mask generation for diffractive optical elements using microcomputers," Appl. Opt. 32(14), 2566-2572 (1993).

5. T. J. Suleski and D. C. O'Shea, "Fidelity of PostScript-generated masks for diffractive optics fabrication," Appl. Opt. 34(4), 627-635 (1995).

6. R. B. Collier, C. B. Burckhardt, and L. H. Lin, Optical Holography, pp. 224-226, Academic Press, London (1971).

7. V. A. Vanin, "Hologram copying (review)," Sov. J. Quantum Electron. 8(7), 809-818 (1978).

8. I. Pascual, A. Beléndez, and A. Fimia, "A two step method for recording holographic optical elements with partially coherent light,"' $J$. Opt. 22, 135-139 (1991).

9. I. Pascual, A. Beléndez, and A. Fimia, "Holographic system for copying holograms by using partially coherent light," Appl. Opt. 31(17), 3312-3319 (1992)

10. J. Campos, B. Janowska-Dmoch, K. Styczynski, K. ChalasinskaMacukow, F. Turon, and M. J. Yzuel, "Computer-generated binary phase-only filters with enhanced light efficiency recorded in silver halide sensitized gelatin," Pure Appl. Opt. 2, 595-605 (1993).

11. H. I. Bjelkhagen, Silver Halide Recording Materials, Springer-Verlag, Heidelberg (1993)

12. D. Casasent, "Computer generated holograms in pattern recognition: a review," Opt. Eng. 24, 724-730 (1985).

13. C. B. Burckhardt, "A simplification of Lee's method of generating holograms by computer," Appl. Opt. 9(8), 1949 (1970).

14. O. Bryngdal and F. Wyrowski, "Digital holography-computer generated holograms," in Progress in Optics, Vol. XXVIII, E. Wolf, Ed., pp. 3-86, Elsevier, Amsterdam (1990).

15. Linotronic 630 is made by Linotype-Hell Co., 425 Oser Ave., Hauppage, NY 11788.

16. J. Crespo, A. Fimia, and J. A. Quintana, "Fixation-free methods in bleached reflection holography," Appl. Opt. 25(10), 1642-1645 (1986).

17. A. Fimia, L. Carretero, R. Fuentes, and A. Belendez, "Noise sources in silver halide volume diffuse-object holograms," Opt. Eng. 34(4), 1108-1115 (1995)

18. L. B. Lesem, P. M. Hirsch, and J. A. Jordan Jr. "The kinoform: a new wavefront reconstruction device," IBM J. Res. Dev. 13, 150-155 (1969).

Andrés Márquez obtained his BS and MSc degrees in physics in the Autonomous University of Barcelona (Spain) in 1996 and 1997 respectively. $\mathrm{He}$ is a $\mathrm{PhD}$ student at the Autonomous University of Barcelona. His research interests include computer-generated holograms, holographic recording materials, and optical processing.

Juan Campos obtained the MSc degree in physics at the University of Zaragoza (Spain) in 1981, and the PhD degree in physics at the Autonomous University of Barcelona (Spain) in 1986. From 1982 until 1993 he was a professor of optics at the University of Barcelona. Since 1993 he has been a professor of optics at the Autonomous University of Barcelona. He has worked in the field of image quality evaluation, dealing with the influence of aberration apodization. He has also been working on optical pattern recognition of color images and designing different kinds of filters by means of computer-generated holograms. He is a member of the SPIE, OSA, EOS, and SEDO.

María J. Yzuel obtained the MSc and PhD degrees in physics from the University of Zaragoza (Spain) in 1962 and 1966, respectively. She has been a professor of optics at the Universities of Zaragoza and Granada, and since 1983 she has been a full professor at the Autonomous University of Barcelona. She has worked in the fields of diffraction image theory and image quality evaluation. She has also worked in optical pattern recognition. She is a Fellow of the OSA. She is a member of SPIE, EOS, and SEDO. She was the president of the Spanish Optical Society from 1993 to 1996. She 
Márquez et al.: Production of computer-generated phase holograms ...

was a vice president of the International Commission of Optics from 1990 to 1996. She was the secretary general of the European Optical Society from 1996 to 1998.

Inmaculada Pascual received her MSc degree in physics from the University of Granada (Spain) in 1986, and her PhD degree in physics from the University of Valencia (Spain) in 1990. Since 1986 she has been a professor of optics at the University of Alicante (Spain). She has worked in the field of holographic recording materials, dealing with photographic emulsions, photoresists, dichromated gelatin and photopolymers, holographic optical elements, hologram copying, and intraocular lenses. She is a member of the Spanish Optical Society, the European Optical Society, and the Holography Working Group of SPIE.

Antonio Fimia received his $\mathrm{PhD}$ degree in physics from the University of Alicante (Spain) in 1982. He was a member of the Departamento Interuniversitario de Optica at the University of Alicante from 1987 until 1997. Since 1997 he has been a board member of the University Miguel Hernández. He has worked in holographic record- ing materials, dealing with silver halide emulsions, bleached emulsions, silver-halide-sensitized gelatin, photopolymers, photoresists, and dichromated gelatin. He has also been working on holographic optical elements, computer-generated holograms, holographic interferometry, and intraocular lenses. $\mathrm{He}$ is a member of OSA, EOS, ARVO, SPIE (Holographic Group), and SEDO.

Augusto Beléndez obtained his graduate degree in physics in 1986 from the University of Valencia (Spain), and his MSc and PhD degrees in physics from the same university in 1988 and 1990, respectively. From 1986 to 1991 he was a professor of applied physics with the Technical University of Valencia, and since 1991 he has been a professor of applied physics at the University of Alicante, Spain. He has worked in the fields of holographic recording materials, holographic optical elements, diffraction theory, and image quality evaluation, dealing with the influence of aberrations on holographic optical elements. Since 1993 he has been the head of the Department of Physics, Systems Engineering, and Signal Theory at the University of Alicante. He is a member of the Spanish Optical Society, European Optical Society, IEEE, OSA, and SPIE. 